Präsentation bei der Jahrestagung der Sektion Methoden der empirischen Sozialforschung 7. und 8. 3. 2008, Bonn.

Schwind, H.-D., Fetchenhauer, D., Ahlborn, W., Weiß, R. (2001): Kriminalitätsphänomene im Langzeitvergleich am Beispiel einer deutschen Großstadt. Bochum 1975 -1986 - 1998. BKA-Reihe Polizei und Forschung Bd. 3, Neuwied.

Streng, F. (2006): Sanktionseinstellungen bei JuraStudenten im Wandel. In: Soziale Probleme, 17 (2), S. 210-231.

Tonry, M. (Hrsg.) (2007): Crime, Punishment, and Politics in Comparative Perspective. Chicago/London.

Tourangeau, R., Rips, L.J., Rasinski, K. (2000): The Psychology of Survey Response. Cambridge.

\section{Fußnoten}

$1 \mathrm{Ob}$ man diese angelsächsischen Befunde auf andere Ländern generalisieren kann, wie dies manche Autoren getan haben, ist freilich ungewiss: schließlich ist die Strafpraxis in Großbritannien im Vergleich zu anderen europäischen Ländern repressiver ausgerichtet (vgl. Tony
2007). Was bedeutet: die Befragten sind in ihrer Orientierung durchaus kongruent mit dem (relativ) repressiven Justizsystem ihres Landes.

2 Eine methodische Variante der Bestimmung von Punitivität wäre hier, sich an der jeweils schwersten Sanktion - etwa „Gefängnis“ - zu orientieren und zum Maßstab der Bestimmung $\mathrm{zu}$ erheben. Eine andere wäre, das gesamte Spektrum möglicher Strafformen, die den Befragten als Antwortmöglichkeiten zur Verfügung stehen, den Graden der Punitivität nach zu skalieren - z.B. von „Freispruch“ bis „Gefängnis“ - und die jeweiligen Skalenwerte der Analyse zugrundezulegen (vgl. z.B. Kesteren 2009, Reuband 2010b).

3 Dass es diese Effekte gibt, darauf verweisen Studien, in denen die Ergebnisse von faceto-face bzw. telefonischen Befragungen mit denen schriftlicher, postalischer Befragungen verglichen wurden. Ihnen zufolge wurde in der schriftlichen Befragungsvariante verstärkt eine punitive Haltung geäußert. Dies konnte bei Fragen zur Todesstrafe belegt werden (Kury 1993: 360, Reuband und Blasius 1996, Reuband 2002: 13, Anm. 2, 2006: 213) ebenso wie bei einer Frage aus dem „International Crime Victim Survey“, bei der es darum ging, wie mit einem jugendlichen Einbrecher umzugehen sei (vgl. Reuband 2010b: 513f., siehe auch Kury 1993: 360, Kury et al. 2002: 32ff.)

4 Andere Befunde zeigt eine Untersuchung von Schwind und Koautoren (2001). Sie bezieht sich jedoch auf eine lokale Studie und erfasste zudem auch nur die Funktion der Gefängnisstrafe, nicht aber die der Strafe für Kriminalität an sich. Angesichts dessen ist die Generalisierbarkeit auf die Bundesrepublik als Ganzes und Strafen hin eingeschränkt.

5 Zwar gingen Mitte der 80er Jahre nur die westdeutschen Studenten in diese Befragungsserie ein und erst nach der Wende auch die ostdeutschen - doch diese Verschiebung in der Zusammensetzung der Stichprobe dürfte für den beschriebenen Wandel nicht verantwortlich sein.

$6 \mathrm{Ob}$ die Stabilität in der Bewertung auch Folge der Tatsache ist, dass ein Fernseher heutzutage preislich günstiger zu erstehen ist als Ende der 80er Jahre - und der Wert des gestohlenen Gutes deshalb niedriger angesetzt wird -, oder ob die Tatsache, dass es sich um einen jugendlichen Täter handelt, zur gleichen Beurteilung geführt hat, oder ob sich generell eine Stabilität der Beurteilung von Kriminalität niederschlägt, ist eine ungeklärte Frage.

\title{
Reformüberlegungen zur Sicherungsverwahrung nach dem Urteil des EGMR in Sachen M. gegen Deutschland
}

Jörg Kinzig, Tübingen

Die Reform der Sicherungsverwahrung, die nicht erst seit dem spektakulären Urteil des Europäischen Gerichtshofs für Menschenrechte (EGMR) in Sachen M. gegen Deutschland ${ }^{1}$ überfällig ist, geht in eine entscheidende Phase. Nachdem das Bundesministerium der Justiz (BMJ) Ende Juli zunächst einen „Diskussionsentwurf Gesetz zur Neuordnung des Rechts der Sicherungsverwahrung und zur Stärkung der Führungsaufsicht" vorgestellt hat, liegt seit Anfang Oktober eine aus dem selben Hause stammende „Formulierungshilfe" vor, die nunmehr den Titel „Gesetz zur Neuordnung des Rechts der Sicherungsverwahrung und zu begleitenden Regelungen “ trägt. Eingang in diese Formulierungshilfe hat in Artikel 5 auch ein „Gesetz zur Therapierung und Unterbringung psychisch gestörter Gewalttäter (Therapieunterbringungsgesetz - ThUG) “ gefunden, mit dem „eine sichere Unterbringung “ derjenigen (ehemaligen) Sicherungsverwahrten ermöglicht werden soll, die infolge des Urteils des EGMR bereits aus der Sicherungsverwahrung in Freiheit entlassen werden mussten oder noch zur Entlassung anstehen. ${ }^{2}$

Im Mittelpunkt meiner folgenden Überlegungen soll aber nicht dieses überaus problematische Vorhaben stehen. Vielmehr hat mich die Redaktion der Neuen Kriminalpolitik (NK) gebeten, auf die von Frommel und Kreuzer im letzten Heft der NK angestellten Reformüberlegungen zu reagieren. ${ }^{3}$

\section{Bedenken gegen die Vorbehaltslösung}

Diskussionsentwurf wie auch Formulierungshilfe sehen einen Ausbau der vorbehaltenen Sicherungsverwahrung in $\$ 66 \mathrm{a}$ StGB vor. ${ }^{4}$ Diesen begrüßen auch Frommel und Kreuzer. ${ }^{5}$

Ich vermag mich dagegen mit diesem Vorhaben nicht anzufreunden. Nur streifen möchte ich bei meiner Kritik die Frage, ob die vorbehaltene Sicherungsverwahrung überhaupt mit den Vorgaben der Europäischen Menschenrechtskonvention (EMRK) in Einklang zu bringen ist. Bekanntlich verfolgt das Konzept der vorbehaltenen Sicherungsverwahrung einen Mittelweg zwischen der traditionellen Sicherungsverwahrung, die zusammen mit der Freiheitsstrafe durch das erkennende Gericht angeordnet wird und der nachträglichen Sicherungsverwahrung, die isoliert am Ende der Freiheitsstrafe erfolgt.

Als Eingriffsgrund für eine vorbehaltene Sicherungsverwahrung dürfte allein Art. 5 Abs. 1 S. 2 lit. a) EMRK in Frage kommen. Dieser verlangt eine „rechtmäßige Freiheitsentziehung nach Verurteilung durch ein zuständiges Gericht“. Ob dieses Erfordernis im Falle einer vorbehaltenen Sicherungsverwahrung gewahrt ist, die vom Schuldspruch abgekoppelt ist, erscheint nach der neueren Straßburger Rechtsprechung jedenfalls nicht unzweifelhaft. ${ }^{6}$ 
Ungeachtet der genannten, für diese Variante der Sicherungsverwahrung geltenden Bedenken, ist nach der Vorgabe der Formulierungshilfe zu trennen zwischen einer vorbehaltenen Sicherungsverwahrung nach $\$ 66$ a Abs. 1 StGB n.F. und einer vorbehaltenen Sicherungsverwahrung für Erst- oder Einmaltäter nach $\ 66$ a Abs. 2 StGB n.F. Letztere halte ich für besonders prekär. $\$ 66 a \mathrm{Abs}$. $2 \mathrm{StGB}$ n.F. soll folgenden Wortlaut erhalten:

„Einen Vorbehalt im Sinne von Absatz 1 kann das Gericht auch aussprechen, wenn

1. jemand zu einer Freibeitsstrafe von mindestens fünf Jahren wegen eines oder mehrerer Verbrechen gegen das Leben, die körperliche Unversehrtheit, die persönliche Freiheit, die sexuelle Selbstbestimmung, nach dem Achtundzwanzigsten Abschnitt oder nach den $\iint 250,251$, auch in Verbindung mit \252 oder $\$ 255$, verurteilt wird,

2. die Voraussetzungen des $\int 66$ nicht erfüllt sind und

3. mit hinreichender Sicherheit feststellbar oder zumindest wahrscheinlich ist, dass die Voraussetzungen des \66 Absatz 1 Satz 1 Nummer 4 vorliegen. "

Die Voraussetzungen des angesprochenen $\ 66$ Abs. 1 S. 1 Nr. 4 StGB n.F. sind dabei zusammengefasst das Vorliegen eines Hangs und eine daraus resultierende Gefährlichkeit.

Nach $\$ 66$ a Abs. 3 S. 2 StGB n.F. ordnet das Gericht die Sicherungsverwahrung endgültig an, „wenn die Gesamtwürdigung des Verurteilten, seiner Tat oder seiner Taten und ergänzend seiner Entwicklung bis zum Zeitpunkt der Entscheidung ergibt, dass von ihm erhebliche Straftaten zu erwarten sind, durch welche die Opfer seelisch oder körperlich schwer geschädigt werden."

Vergleicht man die Anforderungen dieser neuen Sicherungsverwahrungsvariante mit denjenigen der traditionellen Sicherungsverwahrung nach $\ 66$ Abs. $1 \mathrm{StGB}$, die ja fast 30 Jahre lang unangefochten allein das Feld der Sicherungsverwahrung beherrschte, zeigt sich die Auflösung der bei der traditionellen Sicherungsverwahrung in $\$ 66$ Abs. 1 StGB noch bestehenden rechtsstaatlichen Vorkehrungen. Formelle Voraussetzungen wie Vorstrafen und ein Vorvollzug, die zum einen die Ausgestaltung der Sicherungsverwahrung als „ultima ratio“ gewährleisten, zum anderen für eine halbwegs zuverlässige Prognose geradezu unerlässlich sind, fehlen nach $\$$ 66a Abs. 2 StGB n.F. völlig. ${ }^{7}$ Für die Anordnung des Vorbehalts genügt in formeller Sicht die Begehung einer schweren, wenn auch mit einer Freiheitsstrafe von mindestens fünf Jahren abgeurteilten Katalogtat. Dabei kommt in problematischer Weise hinzu, dass auch die Verurteilung zu einer Gesamtstrafe ausreichen kann. ${ }^{8}$

Für eine in Zukunft großzügige Anwendung dieser Variante könnte insbesondere sorgen, dass die Formulierungshilfe bei der endgültigen Anordnung der Sicherungsverwahrung auf das Vorhandensein neuer Tatsachen, sogenannter „Nova“, gänzlich verzichtet. ${ }^{9}$ Dieses Erfordernis hatte aber noch bei der nachträglichen Sicherungsverwahrung eine stark einschränkende Funktion. ${ }^{10}$

Ermittelt man auf Grundlage der Strafverfolgungsstatistik das für einen Vorbehalt nach $\mathbb{S} 66 \mathrm{a}$ Abs. 2 StGB n.F. in Frage kommende Potential an Straftätern, errechnet sich, selbst wenn man die zu lebenslanger Freiheitsstrafe Verurteilten unberücksichtigt lässt, eine Größenordnung von rund 1000 Personen jährlich. ${ }^{11}$ Sollte die vorbehaltene Sicherungsverwahrung in der vorgeschlagenen Form Realität werden, ist daher schon angesichts des derzeit aufgeheizten kriminalpolitischen Klimas zu befürchten, dass die Zahl der Siche- rungsverwahrten weiter, womöglich drastisch, ansteigen wird. Dies würde augenscheinlich im Widerspruch dazu stehen, dass sich auch die Formulierungshilfe zu einer restriktiveren Ausgestaltung der Sicherungsverwahrung zu bekennen scheint. ${ }^{12}$

Darüber hinaus begegnet das Konzept der vorbehaltenen Sicherungsverwahrung denselben grundsätzlichen Bedenken, die die Formulierungshilfe richtigerweise der nachträglichen Sicherungsverwahrung entgegenhält. ${ }^{13}$ So besteht nach der Neukonzeption, wie erwähnt, die Gefahr, dass ein erheblicher Anteil an Vorbehalten ausgesprochen werden wird. Einziger Unterschied zur jetzt bei der nachträglichen Sicherungsverwahrung beklagten Praxis wäre dann der Umstand, dass bei der nachträglichen Sicherungsverwahrung eine Fülle von Straftätern unter einem gesetzlichen, bei der neuen vorbehaltenen Sicherungsverwahrung unter einem gerichtlichen Vorbehalt stehen. Käme es zu dem befürchteten häufigen Ausspruch von Vorbehalten, könnte dies zwei Effekte auslösen:

- Aus einer großen Zahl dieser Vorbehalte dürften am Ende in einer öffentlichen, d.h. unter großem medialen Druck geführten Hauptverhandlung echte Sicherungsverwahrungen werden. Damit würde das Konzept der Sicherungsverwahrung als „ultima ratio" noch weiter aufgegeben.

- Auch wenn die Vorbehalte nicht zu endgültigen Sicherungsverwahrungen erstarkten, gäbe es ein Problem. Denn dann müssten Personen voraussichtlich relativ kurzfristig aus der Freiheitsstrafe entlassen werden, die darauf nicht hinreichend vorbereitet werden konnten.

Ganz generell ist zu erwarten, dass die Strafvollzugsbehören nach einem Vorbehalt wie schon jetzt bei anstehender oder potentiell nachträglicher Sicherungsverwahrung nur selten Vollzugslockerungen gewähren werden. Auch ist zu befürchten, dass sich die von einem Vorbehalt Betroffenen nicht in einem hinreichenden Maße und mit aller Offenheit auf Therapieangebote einlassen werden. Schließlich droht in jedem Fall, dass die dabei gewonnenen Informationen für die ausstehende endgültige Entscheidung über die Sicherungserwahrung Verwendung finden. Dies lässt auch eine erhebliche Belastung des Vollzugsklimas befürchten.

\section{Das Schicksal des Hanges}

\$66a Abs. 2 StGB n.F. führt auch zum Novum einer Sicherungsverwahrung ohne Hang. ${ }^{14}$ So kann die Anordnung des Vorbehalts bei dieser Variante schon dann erfolgen, wenn der Hang nur „wahrscheinlich “ ist. Für die endgültige Anordnung der Sicherungsverwahrung reicht dann nach $\$ 66$ a Abs. 3 S. 2 StGB n.F., wie gesehen, eine reine Gefährlichkeitsprognose aus. $\$ 67 \mathrm{~d}$ Abs. 3 S. 1 StGB, der bisher noch für eine Dauer der Sicherungsverwahrung über zehn Jahre hinaus verlangt, dass "nicht die Gefahr besteht, dass der Untergebrachte infolge seines Hanges erhebliche Straftaten begehen wird“, soll nach der Formulierungshilfe dadurch harmonisiert werden, dass dort die Wörter „infolge seines Hanges“ gestrichen werden.

Was ist von einer solchen neuen Sicherungsverwahrung ohne Hang zu halten? Frommel kritisiert in ihrem Beitrag zunächst völlig zu Recht die gesetzgeberische Konstruktion der Wahrscheinlichkeit einer Hangtäterschaft („absurd“), um für eine Sicherungsverwahrung ohne Hang zu votieren. ${ }^{15}$ Kreuzer/Bartsch lassen das Schicksal des Hangs bei ihrem Vorschlag offen bzw. den Hang bei Ersttätern für „kaum feststellbar“. ${ }^{16}$ 
Tatsächlich haben verschiedene empirische Studien gezeigt, dass das Kriterium des Hangs, ungeachtet seiner fragwürdigen inhaltlichen Überzeugungskraft und historischen Herkunft, ${ }^{17}$ in der Vergangenheit in der gerichtlichen Praxis keine eigenständige Bedeutung aufwies. Denn bei der traditionellen Sicherungsverwahrung nach $\$ 66$ Abs. 1 und 2 StGB wurde das Merkmal des Hangs im Wesentlichen mit Art und Zahl der Vortaten und Vorstrafen und damit fast immer mit solchen Kriterien begründet, die schon Bestandteil der formellen Voraussetzungen waren. ${ }^{18}$

Jedoch ist die rechtstatsächliche Bedeutung des Merkmals des Hanges dadurch gewachsen, dass der Gesetzgeber, beginnend im Jahr 1998, die formellen Voraussetzungen für die Anordnung der verschiedenen Formen der Sicherungsverwahrung sukzessive abgeschliffen hat. ${ }^{19}$ Dies liegt daran, dass als indiziell für das Vorliegen eines Hanges am ehesten eine hohe Anzahl an Vorstrafen und Vortaten, deren Einschlägigkeit, lange Vorverbüßungszeiten, ein (wiederholter) Bewährungsbruch, eine hohe Rückfallfrequenz, ein schneller zur Anlasstat führender Rückfall, eine sich steigernde Schwere der Straftaten sowie ein hoher Spezialisierungsgrad anzusehen sind. ${ }^{20}$ Damit führt das Festhalten am Erfordernis des Hangs dazu, dass damit wenigstens ein Teil der in der letzten Zeit für die Anordnung der Sicherungsverwahrung weggefallenen formellen Voraussetzungen kompensiert wird. Somit trägt das Kriterium des Hangs, auch wenn es fragwürdig ist, dazu bei, die Anwendung der Sicherungsverwahrung wenigstens auf dieser Ebene etwas einzugrenzen. ${ }^{21}$ Im Gegensatz dazu ließe die Aufgabe auch des Hang-Erfordernisses eine weitere Entgrenzung der Sicherungsverwahrung befürchten. Freilich fußen diese Überlegungen auf dem kriminalpolitischen Bekenntnis, die Sicherungsverwahrung müsse „ultima ratio“ des Strafrechts bleiben (oder wieder werden).

\section{Sicherungsverwahrung bei Jugendlichen und Heranwachsenden}

Für die Sicherungsverwahrung gegenüber (ehemals) Jugendlichen oder Heranwachsenden, in welcher Gestalt auch immer, sieht die Formulierungshilfe keine besonderen Regelungen vor. ${ }^{22}$ Es bliebe also (zunächst?) bei den Möglichkeiten einer nachträglichen Sicherungsverwahrung nach den $\$ \int 7$ Abs. 2-4 JGG, 105 Abs. 1 JGG sowie einer vorbehaltenen und nachträglichen Sicherungsverwahrung nach $\ 106$ Abs. 3-7 JGG.

Kreuzer ist der Auffassung, dass auch jugendliche Täter von Gewaltund Sexualstraftaten „ausnahmsweise“ in die vorbehaltene Sicherungsverwahrung einzubeziehen seien, „wenn die Verhältnismäßigkeit zur Schwere der Anlasstat und Strafhöhe gewahrt bleibt und die Tat für die Störung und Wiederholungsgefahr symptomatisch ist." 23

Demgegenüber sollte, wie dies bis zum Jahre 2004 über Jahrzehnte unangefochten der Fall gewesen ist, gegenüber Jugendlichen und Heranwachsenden auf jede Art von Sicherungsverwahrung verzichtet werden. Der nachträglichen Sicherungsverwahrung nach $\mathbb{\$} \mathbb{} 7$ Abs. 2-4, 105 Abs. 1 sowie $\ 106$ Abs. 5-7 JGG ist nach dem Urteil des EGMR in Sachen M. gegen Deutschland ohnehin der Boden entzogen. Die traditionelle oder primäre Sicherungsverwahrung ist schon wegen der damit verbundenen Prognoseschwierigkeiten bisher im JGG nicht eingeführt worden. Aber auch für die vorbehaltene Sicherungsverwahrung gelten die selben Bedenken, die dieser Sanktion bei Erwachsenen entgegengebracht werden. Mehr noch: Eine zuverlässige Kriminalprognose zu erstellen, ist bei nach Jugendstrafrecht Verurteilten sogar noch weitaus schwieriger. ${ }^{24}$
Problematischen Fällen ist am ehesten durch frühzeitig einsetzende (therapeutische) Bemühungen während des Jugendstrafvollzuges, eine sorgfältige Entlassungsvorbereitung und durch eine sinnvoll ausgestalte Führungsaufsicht zu begegnen. Hier wäre es wünschenswert, dass die Neuerungen der im Jahre 2007 ja umfassend reformierten Führungsaufsicht auch in geeigneter Weise implementiert und evaluiert werden. ${ }^{25}$ Auch wenn die hier nur skizzierten Maßnahmen selbstverständlich keine vollständige Sicherheit gewährleisten können, dürften sie für Jugendliche, aber auch Heranwachsende sinnvoller sein als ein Erhalt oder weiterer Ausbau der Sicherungsverwahrung.

\section{Der Autor ist Professor für Strafrecht und Kriminologie der Univer- sität Tübingen.}

\section{Fußnoten}

1 M. gegen Deutschland (19359/04), Urt. v. 17.12.2009.

2 Formulierungshilfe des Bundesministeriums der Justiz „Gesetz zur Neuordnung des Rechts der Sicherungsverwahrung und zu begleitenden Regelungen“", S. 23.

3 Frommel, NK 3/2010, 82 sowie Kreuzer, NK 3/2010, 89.

4 Diesen Ausbau strebt die Formulierungshilfe neben der „Konsolidierung“ der traditionellen Sicherungsverwahrung nach $\$ 66 \mathrm{StGB}$ an, vgl. Formulierungshilfe, S. 2.

5 Frommel, NK 3/2010, 82 (83) sowie Kreuzer, NK 3/2010, 89 (93 ff.).

6 Skeptisch in neuerer Zeit auch: Pollähne, KJ 2010, 255 (264 f.); Eschelbach, NJW 2010, 2499 (2500); einschränkend unter Verweis auf \ 27 JGG: Klesczewski, HRRS 2010, 394 (402); vgl. auch die vorsichtige Formulierung von Fischer, StGB, 57. Aufl. 2010, $\mathbb{S 6 6 a}$ Rdnr. 2: „Dass die Anordnung langjährigen Freiheitsentzugs noch als Rechtsfolge gelten kann, welche ,aus Anlass` einer bestimmten Straftat verhängt wird, wenn sie aufgrund ,der Entwicklung während des Strafvollzuges` erfolgt, ist nicht unzweifelhaft.“ Aus dem ausländischen Schrifttum etwa Leblois-Happe AJPénal 2010, 129 ff.: „Il paraît plus périlleux d'affirmer, sur ce point, la compatibilité avec les dispositions conventionnelles de l'ajournement de l'internement et de la rétention de sûreté du droit français“, wobei bei sie mit dem „l'ajournement de l'internement" explizit auf die vorbehaltene Sicherungsverwahrung Bezug nimmt.

7 Wenn es in der Formulierungshilfe auf S. 41 heißt, die Nummern 1 und 2 enthielten die formellen Voraussetzungen, erscheint dies deswegen eigenartig, weil sich die Nummer 2 des $\$$ 66a Abs. 2 StGB n. F. im Wesentlichen darin erschöpft festzustellen, dass keine (weiteren) formellen Voraussetzungen erforderlich sind.

8 Formulierungshilfe, S. 42.

9 Formulierungshilfe, S. 48.

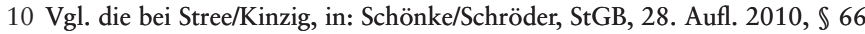
Rdnr. 5 ff. dokumentierte Rechtsprechung.

11 Nach der Strafverfolgungsstatistik 2008 wurden in diesem Jahr allein 1087 Personen wegen einer der in $\ 66$ a Abs. 2 Nr. 1 StGB n.F. genannten Straftaten zu einer zeitigen Freiheitsstrafe von fünf oder mehr Jahren verurteilt.

12 Formulierungshilfe, S. 35.

13 Formulierungshilfe, S. $51 \mathrm{f}$

14 Freilich ist nach Ansicht des 1. Strafsenats des BGH bei der nachträglichen Sicherungsverwahrung nach Jugendstrafrecht ( $\mathbb{7} 7$ Abs. 2-4 JGG) ebenfalls kein Hang erforderlich (vgl. BGH JZ 2010, 683; anders aber das LG Berlin NStZ 2010, 96 (97)). Die Entscheidung des BGH hat (nicht nur bezüglich des Hang-Erfordernisses) einhellige Kritik erfahren (vgl. Bartsch, StV 2010, 521, Eisenberg, JR 2010, 314, Kinzig, JZ 2010, 689, Kreuzer, NStZ 2010, 473, Renzikowski, NStZ 2010, 506).

15 Frommel, NK 3/2010, 82 (87).

16 Kreuzer/Bartsch, GA 2008, 655 (664). Bartsch (Sicherungsverwahrung - Recht, Vollzug, aktuelle Probleme, 2010, 342 f.) spricht sich allerdings dafür aus, dass eine „hangbedingte Gefährlichkeit“ zumindest im Urteil festgestellt werden muss.

17 Dazu Frommel NK 3/2010, 82 (86 f.).

18 Ausführlich Kinzig, NStZ 1998, 14 ff.; vgl. auch Puhlmann/Habermeyer, Forens Psychiatr Psychol Kriminol 2010, 39 ff.

19 Dazu Kinzig, Die Legalbewährung gefährlicher Rückfalltäter (erscheint 2010 in 2. Aufl.).

20 Vgl. Stree/Kinzig, in: Schönke/Schröder, StGB, 28. Aufl. 2010, $\$ 66$ Rdnr. 26 m.w.N.

21 In dieser Richtung auch Bartsch a.a.O., S. 60 ff., 342. Stattdessen enge formelle Voraussetzungen der $\mathbb{\$} 666,66$ a StGB wieder einzuführen (so Frommel NK 3/2010, 82 (85)), wäre zwar wünschenswert, scheint mir aber derzeit kriminalpolitisch utopisch. 
22 Insoweit erfolgen lediglich Anpassungen der bereits vorhandenen Normen. Im Diskussionsentwurf war noch eine Passage enthalten (S. 12), dass die Einbeziehung der Regelungen des JGG erst sinnvoll erscheine, wenn die Diskussion zum allgemeinen Strafrecht abgeschlossen sei. 23 Kreuzer, NK 3/2010, 89 (94).
24 Ablehnend aus der neueren jugendstrafrechtlichen Literatur etwa auch Streng, Jugendstrafrecht, 2. Aufl. 2008, 13 Rdnr. 555-558; Eisenberg, JGG, 14. Aufl. 2010, \106 Rdnr. 4 ff.

25 Z.B. die Möglichkeiten einer Vorstellungs- und Therapieweisung unter Mitwirkung forensischer Ambulanzen (vgl. $\mathbb{S} \mathbb{S} 68$ b Abs. 1 S. 1 Nr. 11, Abs. 2 S. 2 und 3 StGB).

\section{Kirstin Drenkhahn zu Legalbewährung nach Sicherungs- verwahrung}

Rezension zu Jörg Kinzig: Die Legalbewährung gefährlicher Rückfalltäter - Zugleich ein Beitrag zur Entwicklung des Rechts der Sicherungsverwahrung. Berlin: Duncker \& Humblot 2008, XVIII, 348 S., $35,-€$.

Mit „Die Legalbewährung gefährlicher Rückfalltäter“ hat Jörg Kin$z i g$ eine Art Fortsetzung seiner Dissertation (Die Sicherungsverwahrung auf dem Prüfstand, 1996) geschrieben. Seit dem ersten Buch hat sich die Situation grundlegend geändert: Während die Sicherungsverwahrung Mitte der 1990er Jahre im Sanktionensystem kaum eine Rolle mehr spielte (31.3.1996: 176 Verwahrte, 1996 insgesamt 45 neue Anordnungen), scheint sie mittlerweile als kriminalpolitisches Allheilmittel für schwere Delinquenz angesehen zu werden. Seit 1998 wurden nicht nur die Anordnungsmöglichkeiten stetig erweitert, sondern es stiegen auch die Anordnungszahlen (2008: $111 \mathrm{An}$ ordnungen) und die Zahl der Verwahrten (31.8.2009: 500). Zwar machen die Sicherungsverwahrten nur einen geringen Teil der Strafvollzugspopulation (31.8.2009: 72.043) aus, allerdings gibt es bei dieser Gruppe besondere Probleme: Sie müssen getrennt von Strafgefangenen untergebracht werden, es muss ein spürbarer Abstand zum allgemeinen Strafvollzug gewahrt bleiben und der Vollzug muss „die Voraussetzungen für ein verantwortliches Leben in Freiheit [...] schaffen" (BVerfG NJW 2004, 739 [740]), während die Chance auf eine frühzeitige Entlassung sinkt (vgl. Bartsch, Verfassungsgerichtlicher Anspruch und Vollzugswirklichkeit, ZIS 2008, 280 ff.). Empirische Untersuchungen zu dieser Sanktion sind also dringend nötig.

Kinzig hat sein Buch in vier Teile gegliedert. Im ersten Teil zeichnet er auf ungefähr 100 Seiten die Entwicklung der Gesetzeslage und der Rechtsprechung zur Sicherungsverwahrung seit Mitte der 1990er Jahre nach. Die Gesetzgebungsgeschichte wird in fünf Phasen dargestellt, die bestimmte Eskalationsstufen markieren: Die erste Phase ist die Entwicklung bis einschließlich des Gesetzes zur Bekämpfung von Sexualdelikten und anderen gefährlichen Straftaten von 1998 mit der Erweiterung von $\$ 66$ StGB um eine neue Möglichkeit der fakultativen Anordnung von Sicherungsverwahrung bei Verbrechen und bestimmten Vergehen gegen die sexuelle Selbstbestimmung (Abs. 3), mit dem Wegfall der 10-Jahres-Grenze bei der ersten Sicherungsverwahrung und der Änderung der Voraussetzungen der weiteren Vollstreckung freiheitsentziehender Maßregeln. Als zweite Phase werden die landesrechtlichen sog. „Unterbringungsgesetze“, also die Vorläufer der nachträglichen Sicherungsverwahrung, sowie die Einführung der vorbehaltenen Sicherungsverwahrung nach $\$$ 66a StGB besprochen. Die Bilanz dieser Landesgesetze, von denen das erste Anfang 2001 in Baden-Württemberg erlassen wurde, fällt ernüchternd aus, da kaum jemand aufgrund dieser Gesetze untergebracht wurde. Die dritte Phase stellt die Einführung der vorbehaltenen Sicherungsverwahrung auf Heranwachsende, bei denen das allgemeine Strafrecht angewendet wird (\$106 Abs. 3 und 4 JGG), dar, die im April 2004 in Kraft getreten ist und der Kinzig zu Recht attestiert, sie gebe „dem Rechtsanwender nur schwer lösbare Rätsel auf“ (S. 38).

Die vierte Phase sind dann die beiden Entscheidungen des Bundesverfassungsgerichts im Februar 2004 zum Wegfall der 10-JahresGrenze und zu den landesrechtlichen Unterbringungsgesetzen. Zum Wegfall der 10-Jahres-Grenze gibt es mittlerweile ein Urteil des Europäischen Gerichtshofs für Menschenrechte (M. ./. Deutschland, Urt. v. 17.12.2009), der anders als das BVerfG im selben Fall einen Verstoß gegen Menschenrechte (Art. 5 Abs. 1, Art. 7 Abs. 1 EMRK) feststellt. Die Argumentation des EGMR im Hinblick auf einen Verstoß gegen Art. 5 Abs. 1 EMRK deutet darauf hin, dass bei der im Anschluss an eine Freiheitsstrafe angeordneten nachträglichen Sicherungsverwahrung ebenfalls ein Verstoß gegen Art. 5 EMRK naheliegt, da dann der Freiheitsentzug nicht in einer Verurteilung, die einen Schuldspruch wegen einer Straftat enthält, angeordnet wird.

Mit der Einführung der nachträglichen Sicherungsverwahrung $(\mathbb{S} 66 \mathrm{~b} \mathrm{StGB})$ ist die fünfte Phase erreicht. Kinzig geht nach einer Darstellung der ursprünglichen Regelung von 2004 auf die erste Änderung von 2007 ein, mit der das Erfordernis „neuer Tatsachen“, also von Tatsachen, die erst nach der Verurteilung eingetreten oder bekannt geworden sind, für Fälle aufgegeben wird, in denen aus rechtlichen Gründen die Anordnung originärer Sicherungsverwahrung nach $\$ 66$ StGB nicht möglich war (Probleme in den Ost-Bundesländern wegen verzögertem Inkrafttreten von $\$ 66 \mathrm{StGB}$, Fälle des $\$ 66$ Abs. 3 StGB, vgl. dazu Art. 1a EGStGB idF v. 23.9.1990, 16.6.1995 und 26.1.1998).

Es schließt sich ein Überblick über neuere Gesetzentwürfe zur Sicherungsverwahrung an, von denen bisher - und erst nach Veröffentlichung dieses Buches - nur die Ermöglichung nachträglicher Sicherungsverwahrung bei Anwendung des Jugendstrafrechts Gesetz geworden ist. Gerade diese neue Vorschrift in $\$ 7 \mathrm{JGG}$ markiert $\mathrm{m}$. E. einen Systemwechsel, der allerdings schon mit der Einführung der vorbehaltenen Sicherungsverwahrung in $\$ 106$ JGG begonnen wurde. Zwar ist Sicherungsverwahrung bei jugendlichen und heranwachsenden Straftätern nach $\$ \mathbb{} 7$ Abs. 1, 106 Abs. 3 S. 1 JGG ausgeschlossen, allerdings bezieht sich dies jetzt nur noch auf die originäre Sicherungsverwahrung nach $\$ 66 \mathrm{StGB}$. Dass junge - auch junge erwachsene - Straftäter nicht aufgegeben werden dürfen und besondere Anstrengungen unternommen werden müssen, bevor im Erwachsenenalter Sicherungsverwahrung überhaupt in Betracht kommt, ist eine Idee, die exemplarisch in $\$ \mathbb{} \$ 65 \mathrm{Abs}$. 2, $66 \mathrm{StGB}$ idF des 2. StrRG von 1969 zum Ausdruck kam. Politisch hat sie sich anscheinend überlebt. Kinzig macht dies leider nicht deutlich, aber 